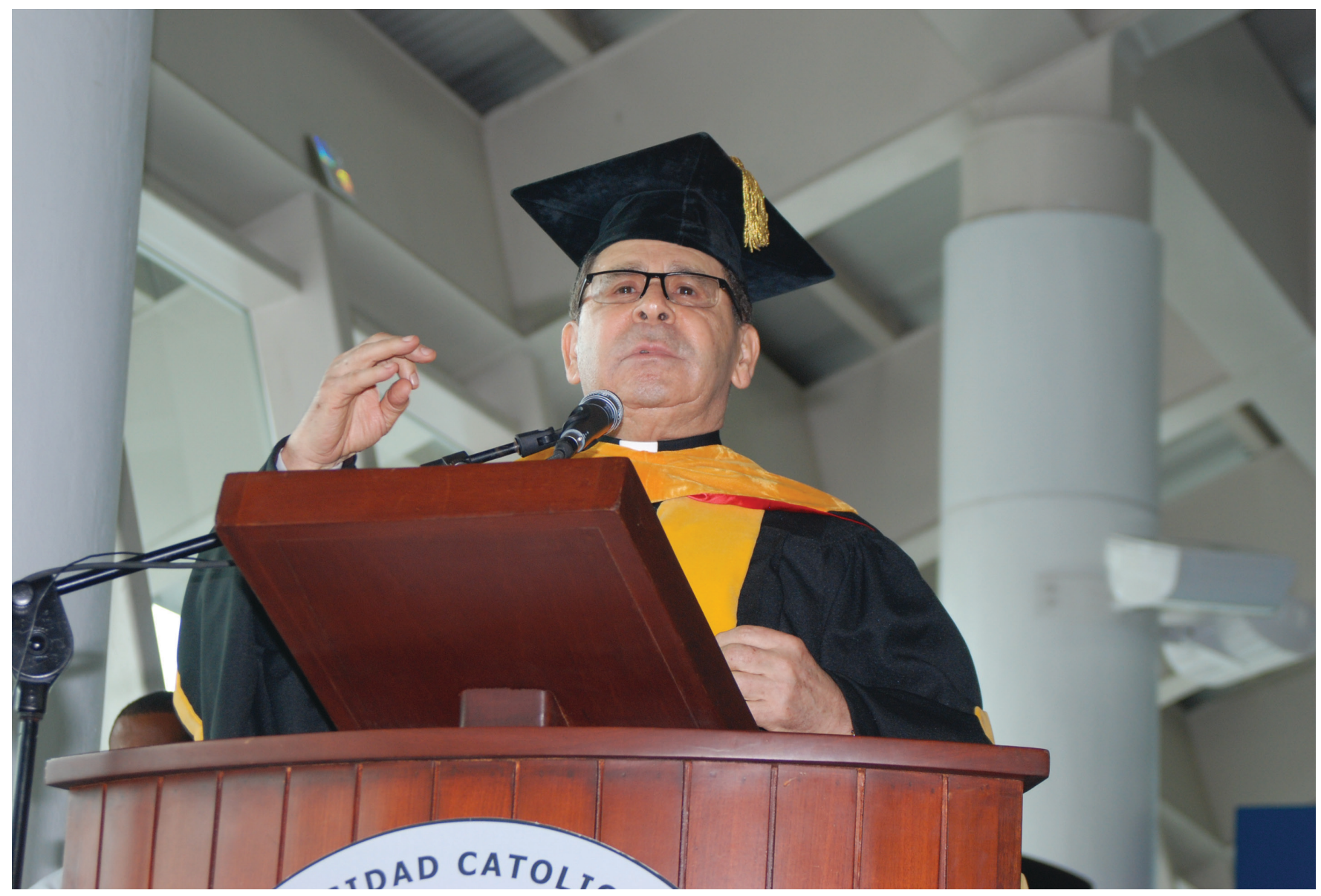

\title{
Cincuenta años de historia de la PUCMM: el rol de monseñor Agripino Núñez Collado
}

in pretender restar méritos a todos los hombres y mujeres que han dedicado parte de su vida a impulsar el desarrollo de la educación superior dominicana, este artículo tiene el propósito de reconocer a uno de los principales ejecutores de la gran obra que es la primera universidad privada de la República Dominicana, la Pontificia Universidad Católica Madre y Maestra. Agripino Núñez Collado, con fe inquebrantable, visión y liderazgo ha estado vinculado, por cinco décadas, a la evolución de la PUCMM. Sus aportes incluso se remontan a la concepción de la idea de su creación junto a Hugo Eduardo Polanco Brito. "Desde los diferentes roles que le ha tocado desempeñar como cofundador, vicerrector y rector, ha impulsado y traducido en realidades concretas y exitosas los principios filosóficos educacionales que dieron origen a la hoy Pontificia Universidad Católica Madre y Maestra" (Oficina de Desarrollo y Comunicaciones. 2005).

En este documento histórico, que contiene algunos testimonios, presentamos de manera resumida la trayectoria académica y la gestión de Agripino Núñez Collado al frente de la PUCMM, así como sus aportes a la educación dominicana, en sentido general. 
Concepción de la hoy Pontificia Universidad Católica Madre y Maestra: un sueño hecho realidad

La idea de crear la hoy Pontificia Universidad Católica Madre y Maestra, surge en un momento histórico en el que el país apenas daba sus primeros pasos hacia un régimen democrático, luego de más de tres décadas de dictadura. Pensar en crear la primera universidad privada, en la segunda ciudad de importancia del país, constituía una utopía, pero no para personas de fe y visionarias como su fundador Hugo Eduardo Polanco Brito y quien fuera uno de sus más cercanos colaboradores, Agripino Núñez Collado, entre otros líderes religiosos, así como emprendedores y generosos munícipes de la ciudad de Santiago.

En el año 1956, siendo aún seminarista, Agripino Núñez Collado fue testigo de encuentros con profesionales y distintos sectores comunitarios convocados por monseñor Polanco, primer obispo de Santiago, con el propósito de intercambiar ideas y conocer las preocupaciones de importantes grupos de la ciudad sobre temas vitales para el desarrollo de la región del Cibao, entre los cuales la educación ocupaba un lugar preponderante.

Habiendo sido previamente profesor y con apenas un año de fundada la Universidad, Agripino Núñez Collado fue nombrado Vicerrector Académico, teniendo que asumir importantes retos, entre los que se encontraban: el escepticismo de muchos que no creyeron posible que la Iglesia fuera capaz de crear una institución educativa de impacto nacional e internacional; la necesidad de expansión física de la Universidad, debido a la creciente demanda y a las nuevas ofertas académicas; la captación de profesionales especializados en las áreas recién creadas; la escasez de recursos económicos para asumir las inversiones necesarias para el desarrollo sostenido de la Universidad y el período de agitación política por el que atravesaba el país, en el que diferentes grupos resurgieron, en busca de espacio en la sociedad y la reivindicación de sus derechos en la naciente democracia.

"Así pues, la Pontificia Universidad Católica Madre y Maestra, nació con una filosofía y unos objetivos bien definidos, que marcaron precisamente un nuevo estilo universitario que se impuso en la República Dominicana con su aparición. Recordemos que al momento del surgimiento de la Madre y Maestra sólo existía en el país la estatal Universidad de Santo Domingo. Varios hechos confirman el nuevo estilo universitario que caracterizó a la Madre y Maestra:

Desde su fundación, se tuvo claro el concepto de que la Universidad ofrecería siempre carreras innovadoras, ligadas al desarrollo, sobre todo, carreras que

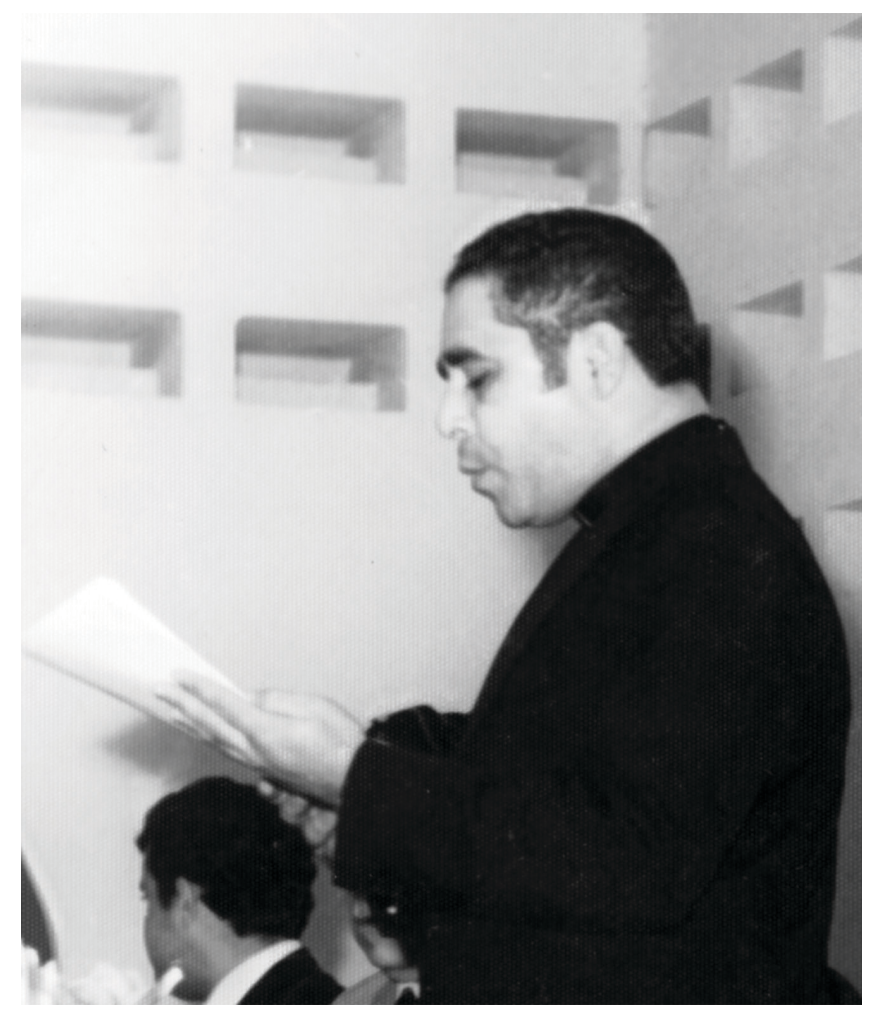

constituyeran una opción nueva y adecuada a la transformación que debía llevar a cabo una sociedad que salía de una tiranía de más de treinta años. Y que los egresados de esas disciplinas se constituyeran ellos mismos en agentes del cambio por todos esperado, en un país que iniciaba su vida democrática.

La Institución tuvo la visión, desde sus primeros años, de abrirse al mundo y tener contacto con las más importantes universidades de Estados Unidos y Europa, con organismos internacionales, y con los sectores público y privado del país, para el desarrollo de sus proyectos. En este contexto, la Institución obtuvo asesorías, presencia de expertos y apoyo financiero para el desarrollo de sus áreas académica y administrativa; esto es, la formación de sus docentes e investigadores, el fortalecimiento de su estructura organizacional así como el desarrollo de su infraestructura física y tecnológica.

La Universidad ha sido, asimismo, pionera en la introducción en la educación superior de la República Dominicana, de procedimientos, normas y criterios educativos, como son:

- La selección de estudiantes por medio de pruebas de aptitud, requisito útil para garantizar la excelencia académica.

- Profesorado de tiempo completo, especializado en su área de docencia.

- El programa de superación del profesorado, hoy Centro de Desarrollo Académico.

- El establecimiento de la carrera académica para el profesorado como una forma de propiciar sentido de pertenencia y permanencia. 


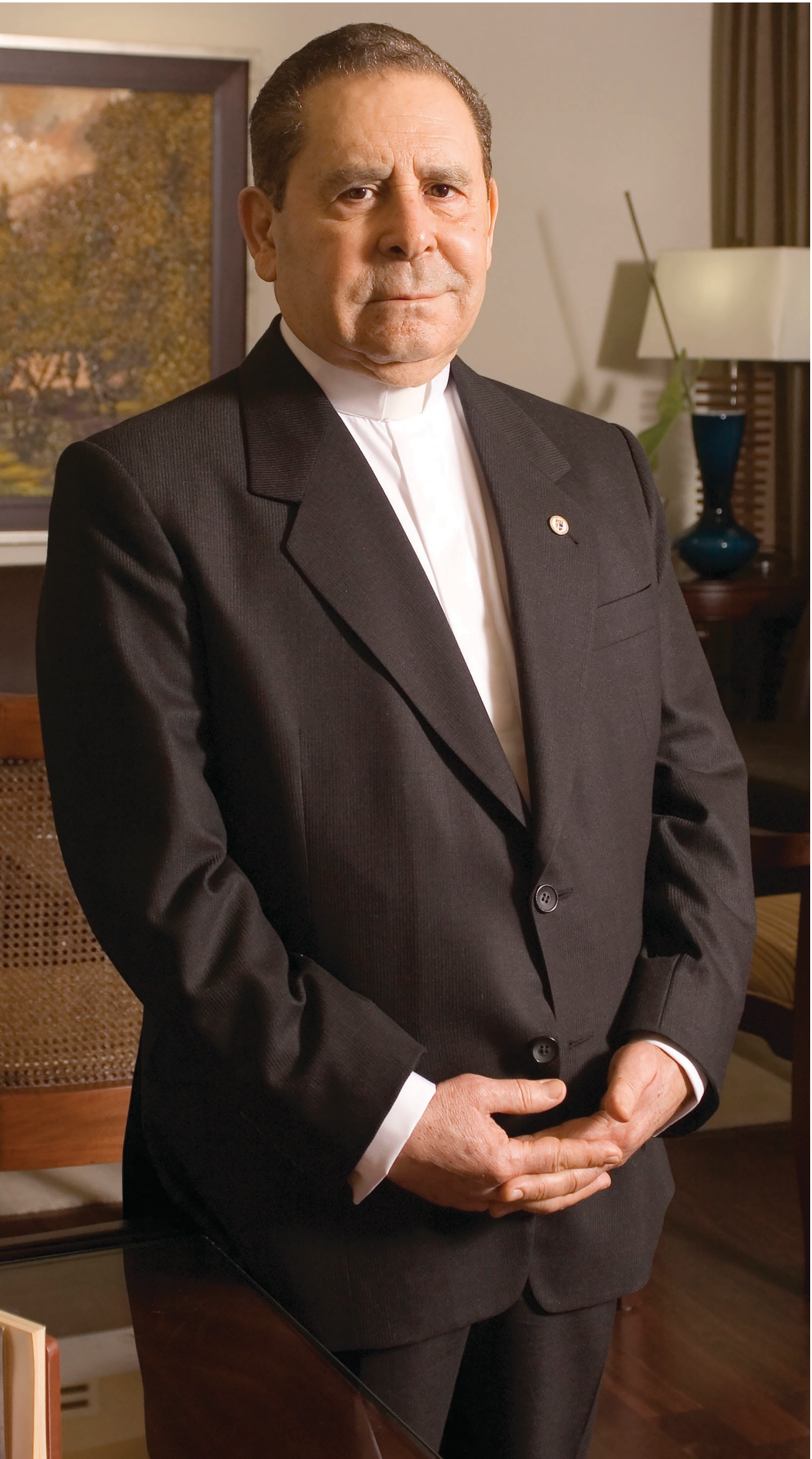

- Sistema de semestre académico y una sesión de verano.

- El Sistema de índice académico para determinar la permanencia del estudiante en la Universidad.

- La departamentalización académica.

- Pasantía obligatoria para algunas carreras que necesitan experiencia práctica.

- Programa de crédito educativo, de su propio presupuesto, para estudiantes con capacidad pero que carecen de recursos económicos, lo que revoluciona el concepto tradicional de que las universidades eran para instrucción de las élites.

- $\quad$ Sistema de pre matrícula, como coadyuvante de la eficiencia en la planificación y administración docente.

- Sistema computarizado para el manejo de la información relativa al estudiante.

- El hecho de que la Institución constituye el lugar de encuentro de todos los sectores de la sociedad, donde se discuten los principales problemas nacionales, en procura de dirimir conflictos y lograr acuerdos importantes para estabilidad política, económica y social del país" (Miriam Cerda).

En otro orden de ideas, tal y como planteaAgripino Núñez Collado en su libro Un Nuevo Estilo Universitario, al terminar la década de los sesenta, la Universidad se convirtió en un centro multicultural, por la presencia de decenas de profesores provenientes de América Latina, Estados Unidos y Europa, los cuales, junto a sus familias, impactaron notablemente la economía y el estilo de vida de la ciudad de Santiago. 
Entre los acontecimientos más sobresalientes del primer decenio de la Universidad y en el cual Agripino Núñez Collado asumió los roles de profesor y Vicerrector Académico, se citan los siguientes:

- La valentía de la Iglesia Católica, sus feligreses y el empresariado de la ciudad de Santiago al iniciar un proyecto novedoso de gran trascendencia, sustentado en la fe y el optimismo.

- La obtención de la personalidad jurídica de la Universidad y su derecho a conferir títulos académicos.

- Dar los primeros pasos para el establecimiento de una estructura académica, compuesta por facultades, con el inicio de la Facultad de Administración e Ingeniería Electromecánica.

- Firma de un convenio de colaboración con la Universidad de Saint Louis, financiado por la Agencia para el Desarrollo Internacional (AID), mediante el cual se obtuvieron fondos para la especialización y adiestramiento del personal docente y administrativo.

- Adquisición de terrenos para la instalación del Campus Universitario, inaugurado el 26 de enero de 1967.

- Haber logrado un mayor involucramiento del sector privado para apoyar un proyecto que crecía a un ritmo superior a las expectativas. De ahí que se conformara una Oficina de Desarrollo y Recaudación de Fondos, con asesoramiento de la Universidad de Saint Louis y contando con un Consejo de Desarrollo.

- Modificación estatutaria que permitió que la Conferencia del Episcopado Dominicano delegara la autoridad para el Gobierno de la Universidad en una Junta de Directores.

- Creación del Decanato de Estudiantes, el cual tiene la responsabilidad de coordinar las actividades cocurriculares y extracurriculares, contribuyendo a la formación integral del estudiantado.

- Promulgación de la Ley 76 de 1965, ley que facilitó el apoyo de organismos internacionales, especialmente del Fondo Especial de las Naciones Unidas y del Banco Interamericano de Desarrollo (BID).

- Creación de las carreras de Trabajo Social, Enfermería a nivel universitario, Administración Pública, Ingeniería Industrial y Economía, siendo pionera en la oferta de dichas carreras. También inició la carrera de Ingeniería Agronómica.

- Diseño de instrumentos necesarios para el funcionamiento interno de la Universidad, como fueron: el Reglamento de Profesores y de Carrera Docente y el Reglamento de Participación Estudiantil. También se oficializaron las Vicerrectorías Académica y de Desarrollo.
En una publicación que recoge el pensamiento universitario latinoamericano se hace la siguiente aseveración: Con siete años como Vicerrector y más de 37 años como Rector, Agripino Núñez Collado es pues el líder por excelencia de esa institución académica.

A Núñez Collado le correspondió resolver el conflicto que fue surgiendo a partir de la guerra civil del 1965 en torno a los reclamos de profesores y estudiantes de mayor participación en el gobierno, definiendo los límites de sus derechos y deberes en una universidad privada. A partir de esa etapa, la UCMM, hoy Pontificia, logró superar esas dificultades y avanzó, con paso firme, hacia el desarrollo institucional y la excelencia académica (Ricart, 2008).

\section{La reflexión colectiva como estrategia para el cambio y el desarrollo académico de la PUCMM}

La auto reflexión y revisión del quehacer de la Universidad ha sido una labor permanente en la historia de vida de la Institución. Agripino Núñez Collado propició que las grandes transformaciones y proyectos de desarrollo de la PUCMM fueran precedidos por exhaustivos procesos de reflexión institucional.

Diversos son los mecanismos que se emplearon para garantizar la participación de la comunidad universitaria en la discusión y búsqueda de alternativas para el desarrollo integral de la Universidad. Contó, además, con el apoyo de organismos y expertos internacionales, con vasta experiencia en las áreas objeto de consulta. Entre los mecanismos podemos citar la conformación de comités, la organización de seminarios-talleres evaluativos, así como reuniones con determinados grupos de interés para el análisis de tópicos puntuales.

En la década de los ochenta se institucionalizaron los encuentros entre la Conferencia del Episcopado Dominicano y la Junta de Directores, con la finalidad de analizar la situación interna de la Universidad y sus retos ante el contexto socioeconómico y educativo nacional e internacional.

Esta es una actividad permanente que propicia un diálogo abierto en un ambiente de camaradería que favorece la integración entre las personas que tienen en sus manos el destino de la Universidad. De igual manera, se da un vistazo a la situación social, económica, educativa y política del país, a fin de determinar la forma en cómo la Universidad puede ser impactada e impactar, aportando soluciones a los problemas fundamentales de la sociedad dominicana, a partir de los principios filosóficos que la sustentan. 
Principales iniciativas para el fortalecimiento académico, el desarrollo físico y la proyección internacional de la PUCMM

El segundo decenio de la Universidad inició con la designación de Agripino Núñez Collado como Rector. A partir de este período, la Universidad tuvo un notable despegue y se fortaleció en sus áreas académica y administrativa.

En materia de captación y visión de desarrollo Agripino Núñez Collado es un verdadero maestro. Siempre ha tenido la capacidad de adecuar las peticiones y necesidades institucionales a la visión y el discurso del donante, sin perder ni un ápice el objetivo institucional. El programa de crédito educativo para los estudiantes es una muestra de esa preocupación.

Los hechos más relevantes en el segundo, tercero y cuarto decenio de la PUCMM son:

- Construcción de la capilla "Nuestra Señora de la Anunciación", creando la primera parroquia universitaria, como centro para la promoción de los valores cristianos. En efecto, se fortalece y amplía la pastoral universitaria y se crea el primer grupo nacional de cursillistas universitarios de cristiandad.

- Primer Préstamo BID, en el año 1971, para la consolidación y ampliación del desarrollo físico y académico de la Universidad.

- Establecimiento de las extensiones de Bonao y Puerto Plata.

- Puesta en ejecución de una nueva estructura académica y administrativa de la Universidad.

- Inauguración del edificio en el que funcionan las unidades de Administración y Servicios Generales y el Centro de Cómputos.

- Creación de la Oficina de Egresados y Pasantes, con el propósito de mantener el vínculo de los egresados con la Universidad y viabilizar su inserción al mercado laboral.

- Inicio de la construcción del Teatro Universitario.

- Inauguración de la Escuela Hotelera.

- Creación de la Facultad de Ciencias de la Salud en el año 1974. Como resultados inmediatos, se establecieron acuerdos con la Secretaría de Estado de Salud Pública y Asistencia Social para integrar los servicios de la Facultad al sistema público de salud. Además, se realizaron las gestiones para que el Gobierno construyera y equipara el Hospital Regional Universitario José María Cabral y Báez.

- Segundo préstamo BID en el 1977.

- Con los aportes del gobierno de Inglaterra, a través de la Universidad de Gales y las empresas mineras establecidas en el país, se creó la carrera de Ingeniería de Minas.
- Por considerar al personal docente como uno de los agentes principales para el logro de la innovación y la excelencia académica, se destinaron fondos para el Programa de Superación del Profesorado.

- Creación, en el año 1977, del Departamento de Educación Continuada y Asistencia Técnica (DECAT), con el propósito de establecer los vínculos entre la Universidad y las organizaciones productivas del país.

- Realización de un "Segundo Seminario de Evaluación y Cambio de la Universidad", que reunió a las principales autoridades académicas y administrativas con el objetivo de revisar el funcionamiento interno a la luz de logros y problemas futuros.

- Establecimiento del Recinto Santo Tomás de Aquino (RSTA), en el año 1981, con el propósito inicial de ofrecer programas de postgrados y maestrías y cursos de alta gerencia en las áreas de Administración Pública, Economía y Turismo, entendidas como prioritarias y que no eran atendidas por ninguna otra institución.

- Con la finalidad de analizar los problemas políticos y sociales y contribuir a la búsqueda de soluciones, se establece en el RSTA, el Centro Universitario de Estudios Políticos y Sociales (CUEPS).

- Proceso de reflexión y análisis sobre el rol que debería desempeñar la Universidad en la próxima década. El mismo fue denominado PLANDIUCA.

- Creación de las carreras de Ingeniería de Sistemas y Computación, Ingeniería Electrónica y de Topografía y Agrimensura, en el campus de Santiago. En Santo Domingo, por su parte, se inicia el Postgrado en Derecho Social.

- Se inicia la organización de la Fundación Damas Amigas, con el propósito de gestionar y proveer recursos para financiar estudios a jóvenes talentosos con limitaciones económicas.

- Creación, en el año 1985, del Centro de Estudios y Servicios Empresariales (CEYSE) y el Centro de Estudios Urbanos y Regionales (CEUR). El primero, asumió parte de los propósitos del DECAT de incidir en el desarrollo económico y social de las instituciones públicas y empresas del sector privado, a través de la profesionalización y actualización de sus recursos humanos. El segundo (CEUR), tiene su misión orientada hacia la investigación, docencia/capacitación, servicios comunitarios y consultorías para el desarrollo sustentable de los asentamientos humanos; luchando contra la pobreza, y por una distribución equitativa de los recursos humanos, tecnológicos y económicos en el desarrollo urbano-regional.

- La Universidad fue erigida canónicamente Católica y Pontificia, mediante decreto No. 1504/86/26, del día 9 de septiembre del año 1987, de la Congregación para la Educación Católica. 
- Establecimiento de planes de seguro de vida, médico y de hospitalización, así como un plan de pensiones y jubilaciones para todo el personal que labora en la Institución.

- La puesta en ejecución de los servicios a la comunidad, a cargo de profesores y estudiantes del área de salud, en centros periféricos de medicina asistencial. Estos servicios benefician a los sectores marginados de la comunidad regional.

- Inauguración de la Parroquia Universitaria Nuestra Señora de la Anunciación.

- Desarrollo de la infraestructura de apoyo tecnológico, dado el ofrecimiento de programas virtuales, videoconferencias y otros programas compartidos con prestigiosas universidades.

- Fortalecimiento del Programa de Superación del Profesorado, a través de una reestructuración organizativa, el ofrecimiento de facilidades para la actualización de los profesores, tanto dentro como fuera del país y el incentivo económico para el desarrollo de investigaciones.

- Crecimiento sostenido de la matrícula estudiantil.
De manera particular, en los programas de postgrados y maestrías.

- Firma de convenios de cooperación con entidades nacionales e internacionales para proyectos académicos especializados y de investigación científica.

- Elaboración del Plan de Desarrollo Estratégico.

- Modificación de la estructura organizativa, trayendo como resultado la unificación de instancias académicas y administrativas para maximizar el aprovechamiento del conocimiento y de las experiencias del talento humano de la Institución.

- Creación de tres vicerrectorías: de Postgrado, de Investigación e Innovación y de Relaciones Internacionales y Cooperación.

- Establecimiento de una Junta Universitaria y un Consejo Académico Colegiado, así como de facultades y otras unidades departamentales académicas y administrativas, respondiendo a una visión integrada de procesos y servicios para la Institución como un todo.

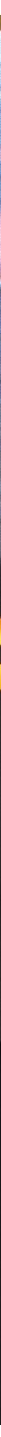


Principales aportes de la PUCMM a la Educación Dominicana

La PUCMM ha asumido como uno de sus principales compromisos contribuir al fortalecimiento de la educación dominicana. De ahí, su experiencia de más de medio siglo en la formación de profesionales en el área de la Educación. Entre otras iniciativas, ha realizado aportes en la formación de maestros en servicio y de nuevos prospectos; ha desarrollado proyectos de investigación-acción que contribuyen a la mejora de la práctica docente y de las competencias de los estudiantes en áreas básicas, como Matemática y Lengua Española.

Diversos convenios y acuerdos de colaboración con organismos nacionales e internacionales han configurado la oferta y el aporte de la Institución en distintas áreas y niveles de la educación dominicana.

A continuación se presenta una sinopsis histórica de esa experiencia:

1962. Surge la Universidad y con ella la Facultad de Educación. En esta facultad se inicia la carrera de Educación para la enseñanza secundaria en las menciones de Administración Escolar y Orientación Escolar.

1971. Inicio a los programas sabatinos para Maestros en Servicio, con el propósito de contribuir al fortalecimiento académico de los docentes que realizaban su ejercicio en las escuelas del sector público.

1972. Se introduce una reforma curricular al plan de estudios de la carrera, con el apoyo de consultores del Banco Interamericano de Desarrollo y de la Universidad de Puerto Rico.

Firma del acuerdo Interuniversitario entre la Secretaría de Estado de Educación, Bellas Artes y Cultos (SEEBAC), la Organización de las Naciones Unidas para la Educación, la Ciencia y la Cultura (UNESCO) y otras universidades para ofrecer programas para la formación de maestros de educación media en las áreas de Matemática-Física, Administración y Supervisión Escolar, Química-Biología, Lengua Española y Literatura, Ciencias Sociales, Orientación Escolar, Lenguas Extranjeras y Ciencias Secretariales.

1973. Acuerdo de colaboración con la SEEBAC y otras universidades nacionales para la formación de profesores en la enseñanza media en la República Dominicana.

1979. Acuerdo de colaboración con el Plan Sierra para llevar a cabo un programa de servicios en el área de educación. Este programa consistió en el entrenamiento de profesores para el mejoramiento de la calidad de vida y conservación de recursos naturales de la zona.

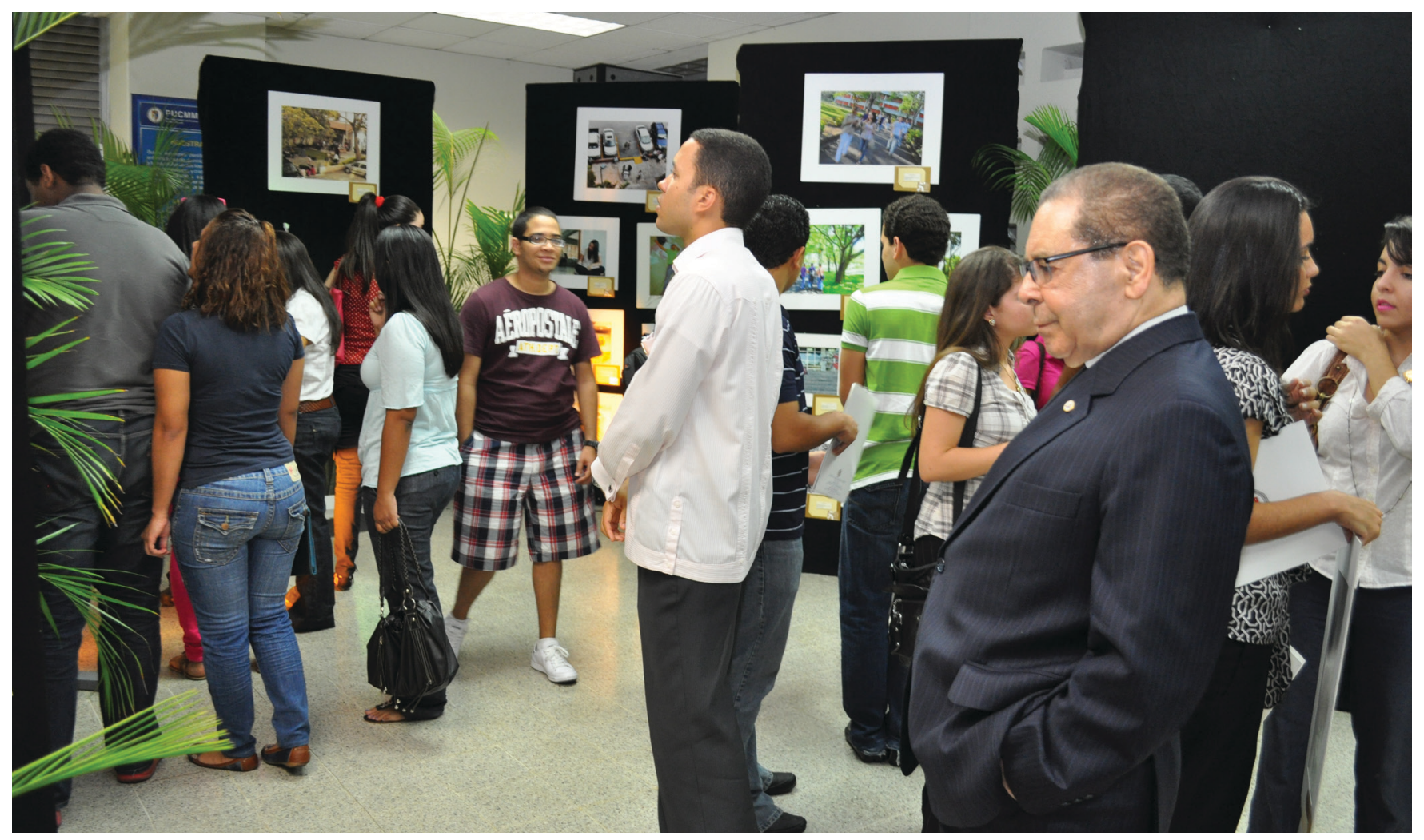

«Cincuenta años de historia de la PUCMM: el rol de monseñor Agripino Núñez Collado» Cuaderno de Pedagogía Universitaria Año 11 / N.21 / enero-junio 2014 / Santiago, República Dominicana / PUCMM / p. 86-97 
1981. Creación del Centro de Documentación e Investigación Educativa (CEDIE), única entidad de este tipo en el Caribe, perteneciente a la Red Latinoamericana de Información y Documentación en Educación (REDUC).

1982. Firma de convenio con el Centro Internacional de Investigaciones para el Desarrollo, con el propósito de investigar el rendimiento académico de los estudiantes de matemática en la República Dominicana.

1984. Proyecto de investigación sobre la aplicación de los microcomputadores en la enseñanza de las ciencias matemáticas, el cual contó con el aval financiero de la UNESCO.

1990. Realización de un programa conjunto con la Secretaría de Estado de Educación Bellas Artes y Cultos para mejorar la enseñanza y aprendizaje del español en las escuelas públicas del país.

Firma de Convenio con la Secretaría de Estado de Educación Bellas Artes y Cultos para el adiestramiento a 50 profesores en metodología de la enseñanza y aprendizaje de la matemática.

1991. Colaboración en la mejoría de la enseñanza y aprendizaje a nivel de la educación primaria y secundaria, con énfasis en la enseñanza del español como lengua materna y de la matemática de las escuelas públicas del país, mediante la elaboración de material didáctico y libros de texto, programas de adiestramiento de profesores y elaboración de programas educativos para radio y televisión, con el propósito de elevar el nivel cultural de la población.

1993. Programa de Capacitación de Maestros en servicio del Nivel Básico para el Proyecto de Desarrollo de la Educación Primaria (PRODEP), con el auspicio del Banco Mundial.

Acuerdo de colaboración con la UNESCO para la medición de niveles de calidad, que sería empleado en la investigación de los niveles de cuarto grado de la educación básica en República Dominicana.

1994. Inicio del Programa de Profesionalización de Maestros y Bachilleres (PPMB). Dicho programa abarcó los niveles de profesorado, licenciatura y maestría, dentro de un acuerdo suscrito con la entonces Secretaría de Estado de Educación y los auspicios del Banco Interamericano de Desarrollo (BID).

Inicio del Proyecto "Tiempo de Lectura", concebido como de colaboración con la educación primaria, promoviendo la formación de lectores críticos en América Latina, a través de un software con textos interesantes, gráficas atractivas e interacciones estimulantes y proveyendo una capacitación adecuada a los avances tecnológicos de la sociedad moderna.

1995. Se inician los programas de maestría mediante un acuerdo en las especialidades de Administración, Supervisión y Gestión de Currículos.

1998. Inicio de programas de postgrados para directivos y técnicos docentes del sistema educativo en las áreas de: Lingüística Aplicada, Ciencias Naturales, Matemática Educativa, Ciencias Sociales y Administración de Centros Educativos. Además, la Maestría en Administración de Centros Educativos.

1999. Se inicia en el RSTA la Maestría en Planificación y Administración de la Educación.

2001. Proyecto Kellogg-PUCMM diseñado para contribuir a que cinco escuelas de las zonas pobres de los sectores urbanos y rurales de la provincia de Santiago de los Caballeros ofrecieran un servicio educativo funcional y de calidad, a través del fortalecimiento de la gestión de los dirigentes y la capacitación de docentes.

2002. Inicio del Programa de los Centros de Excelencia para la Capacitación de Maestros (CETT) en Centroamérica. Este Programa es una iniciativa presentada en la Cumbre de las Américas por el entonces Presidente de los Estados Unidos de Norte América, George W. Bush, a través de la Agencia de los Estados Unidos para el Desarrollo Internacional (USAID). Mediante concurso y luego de un proceso de selección entre más de 50 Universidades, resultaron ganadoras la Universidad del Valle, de Guatemala; Universidad Pedagógica Nacional Francisco Morazán, de Honduras; Instituto Latinoamericano para la Comunicación Educativa (ILCE), de México y la Pontificia Universidad Católica Madre y Maestra, de República Dominicana.

Acuerdo de colaboración con la SEEC, el Consorcio de Educación Cívica para desarrollar dos programas a nivel básico y medio en esta área, con el auspicio del Banco Interamericano de Desarrollo (BID).

Establecimiento de un acuerdo de colaboración con el Grupo E. León Jimenes para desarrollar un programa de postgrado en Educación Cívica.

Como aportes recientes al Sistema Educativo Dominicano y como producto de procesos exitosos de investigación y desarrollo de la Universidad, se 
destacan como otros aportes al Sistema Educativo Dominicano, además de la experiencia en las áreas de Lengua Española y Matemática, los componentes de Gestión y Escuelas Seguras para las intervenciones financiadas por la USAID.

\section{Monseñor Agripino Núñez Collado: Un vistazo a su persona y a su carrera como educador.}

Muchas son las condiciones que conforman la personalidad de monseñor Agripino Núñez Collado, como ser humano, como ciudadano, y a través de sus aportes a la educación superior dominicana.

Uno de los valores que distinguen a monseñor es su inquebrantable fe en Dios. Entre otros testimonios de vida, aún en las peores situaciones económicas, políticas y sociales que han afectado al país, él ha emprendido ambiciosos proyectos, con la esperanza de que Dios abra los caminos para su culminación. Las más importantes iniciativas de la Universidad han sido impulsadas exitosamente en situaciones similares. Sin embargo, no se conforma sólo con creer, sino que sigue las instrucciones del apóstol Santiago que señala que "...la fe, si no produce obras es que está muerta" (Stgo 2, 14-26), por lo que realiza ingentes esfuerzos y gestiones para materializar los proyectos que se propone.

Monseñor Agripino Núñez Collado, en su larga trayectoria de servicio, ha puesto de manifiesto un liderazgo humanístico y visionario, impregnado de fe, firmes convicciones y dinamismo, lo que le ha permitido, en cada escenario de la vida nacional y en diferentes momentos históricos, ser un impulsor, del desarrollo, la institucionalidad y el estado democrático de la República Dominicana (Carmen Pérez Valerio).

En innumerables ocasiones ha expresado su convicción de que la educación es el medio más expedito para alcanzar el desarrollo individual y social, que se constituye como un medio valioso para que el estudiante, futuro profesional, se forme a sí mismo e impacte al desarrollo de la sociedad.

Como auténtico académico, ha entendido la importancia de los actores fundamentales del proceso de enseñanza-aprendizaje, teniendo siempre las puertas de su despacho abiertas para escuchar ideas e inquietudes de profesores y estudiantes. Valora sobremanera al profesor como ente fundamental para el logro de aprendizajes de calidad y como agente transformador de la vida de los educandos. Sus argumentos evidencian la convicción de que el maestro sabe, enseña y ama, a sabiendas de que el amor está por encima del saber y que solo se aprende de verdad cuando se enseña con amor.
Monseñor Agripino Núñez Collado siempre se expresa con notable emoción cuando habla de los egresados, a quienes presenta como lo más importante que pueda exhibir la Institución al servicio de la sociedad. El aprecio del Rector por los ex alumnos ha sido reciprocado por parte de distintas promociones profesionales y de la Asociación de Egresados de la Universidad.

"La PUCMM se ha constituido, bajo su liderazgo intelectual y humano, en un referente nacional de formación profesional de sus egresados, que exhiben un alto estándar ético, un desempeño exitoso en el mundo laboral y en el servicio a la sociedad, con una sólida base formativa respecto de los desafíos que imponen los problemas sociales" (José Darío Suárez Martínez).

Los intereses y vínculos de monseñor Agripino Núnez Collado con la educación, y las humanidades en sentido general, tienen su origen antes de su labor pastoral en la PUCMM y fueron impregnados a través de su formación familiar, académica y por las diferentes responsabilidades que le ha correspondido ejercer. Realizó sus estudios secundarios en Filosofía y Teología en el Seminario Santo Tomás de Aquino, Santo Domingo en el período comprendido entre los años 1948 y 1956. Cursó una licenciatura en Filosofía de la Universidad Autónoma de Santo Domingo, en el 1957 y en Teología y Derecho Canónico, en la Universidad Pontificia de Salamanca, España, en el 1959. Fue profesor del Liceo Secundario Ulises Francisco Espaillat, desde el 1960 hasta el 1962. Obtuvo el grado de Maestría en Administración de la Universidad de Puerto Rico, Recinto Río Piedras, en el 1970.

Las múltiples facetas de la personalidad y la obra de Agripino Núñez Collado se han ido conformando en sus responsabilidades académicas, sus aportes cívicos, así como en los espacios en que ha ejercido su liderazgo académico y social. Sus principales responsabilidades académicas han sido:

- Vicerrector del Colegio Hispanoamericano de Salamanca, España, 1959-1960.

- Director de Obra Diocesana de las Vocaciones, Diócesis de Santiago, 1960-1962.

- Primer Rector del Seminario Menor San Pío X de Santiago de los Caballeros, 1962-1963.

- Vicerrector Académico de la Universidad Católica Madre y Maestra, 1963-1970.

- Rector de la Pontificia Universidad Católica Madre y Maestra, desde 1970 hasta el 2015. 


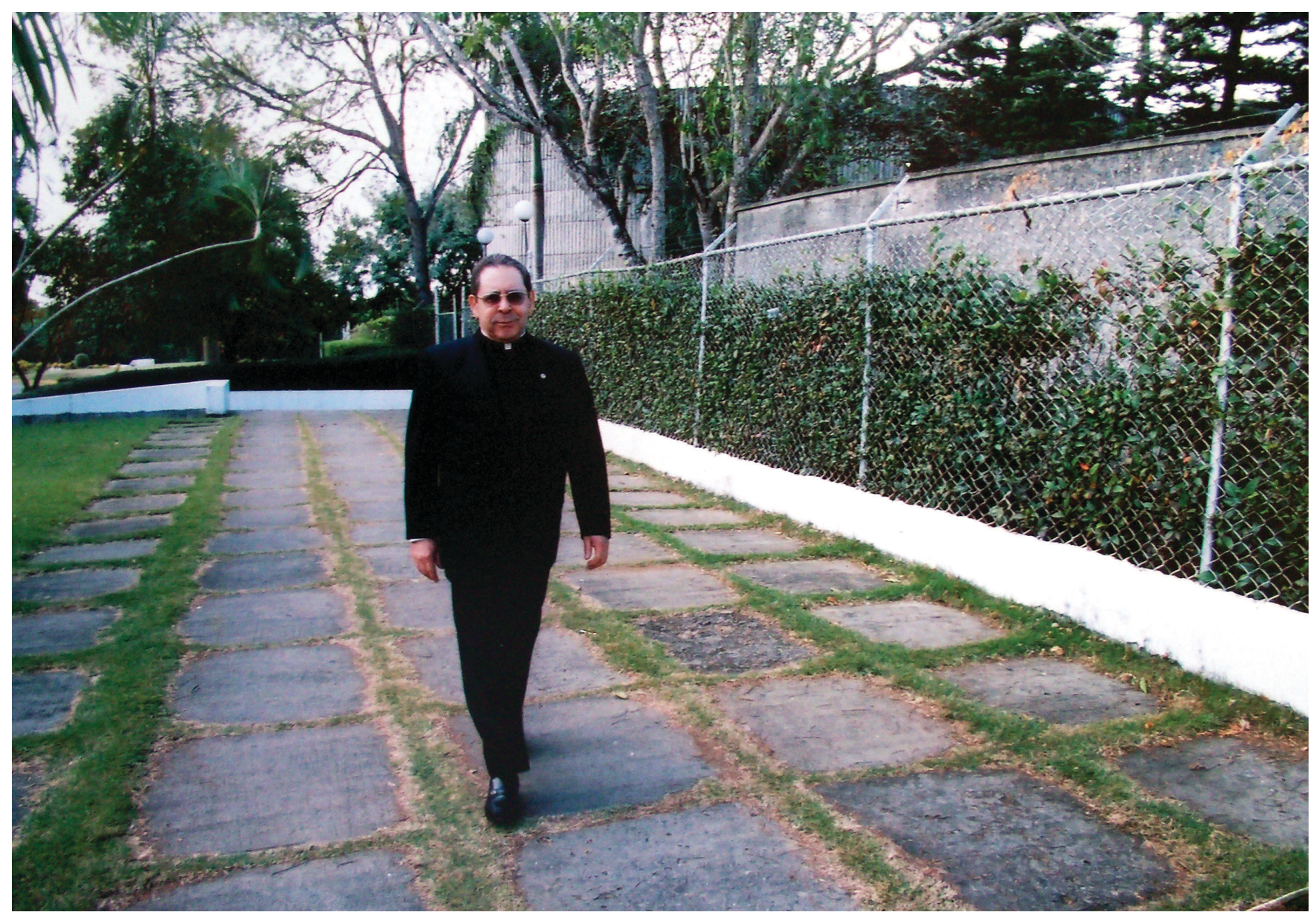

Algunas de las posiciones importantes que ha desempeñado en Organismos Académicos Internacionales son:

- Miembro fundador del Consejo Directivo de la Asociación de Universidades e Institutos de Investigación del Caribe (UNICA), Puerto Rico, 1967.

- Miembro Fundador del Consejo Universitario Interamericano para el Desarrollo Económico y Social (CUIDES), 1982.

- Vicepresidente del Consejo Universitario Interamericano para el Desarrollo Económico y Social (CUIDES), 1982.

- Miembro Fundador del Grupo Universitario Latinoamericano de Estudios para la Reforma y el Perfeccionamiento de la Educación (GULERPE).

- Miembro Fundador y Vicepresidente para la Región del Caribe y Centroamérica de la Organización Universitaria Interamericana (OUI), 1990-1994.

- Vicepresidente de la Federación Internacional de Universidades Católicas-FIUC, 1982-1988.
- Vicepresidente para el Capítulo Centroamericano y del Caribe de la Asociación Internacional de Presidentes y Rectores de Universidades-IAUP, 1993.

- Miembro de la Comisión para el Desarme de la Asociación Internacional de Presidentes y Rectores de Universidades-IAUP/UN, 1993.

- Miembro de la Asociación Universitaria Iberoamericana de Postgrado, desde 1998.

Monseñor es además Presidente del Consejo Económico y Social, organismo consultivo del Poder Ejecutivo en materia económica, social e institucional, que tiene como atribución general conocer y emitir su opinión sobre problemas de cualquier naturaleza de interés nacional, constituyéndose en un espacio institucional permanente de diálogo y deliberación en procura de la concertación social.

\section{Liderazgo Social de monseñor Agripino Núñez Collado}

Es conocido el papel de la Universidad en la creación de una nueva cultura del diálogo y la concertación bajo el liderazgo de monseñor Agripino Núñez Collado, 
así como su presencia activa en la búsqueda de soluciones a las diversas crisis que han afectado a la sociedad dominicana en los últimos tres lustros. De ahí que como educador y conciliador ha procurado el fortalecimiento del sistema democrático del país, por lo que ha interiorizado el diálogo como una vía expedita para la participación y el entendimiento de distintos grupos sociales.

Los enunciados de la misión de la PUCMM referidos a "buscar soluciones científicas a los desafíos que enfrenta el pueblo dominicano y su entorno global... manteniendo el carácter de espacio abierto para la libre discusión de las ideas", han sido honrados por monseñor Agripino Núñez Collado, al ejercer su liderazgo social y capacidad conciliadora en beneficio de los mejores intereses de la Nación.

En efecto, entre las múltiples valoraciones a su persona, por el importante liderazgo como agente de paz y mediación, se afirma que: "A lo largo de su carrera como rector universitario, la labor de Núñez Collado ha sido realmente extraordinaria, tanto para la educación superior como en la mediación y solución de conflictos sociales y políticos por su liderazgo y preocupación por el desarrollo y la preservación del sistema democrático dominicano. Le ha correspondido promover una auténtica cultura del diálogo para la concertación social y política en la República Dominicana, que ha permitido al país avances importantes en la gobernabilidad democrática, lo que se ha redundado en el prestigio del
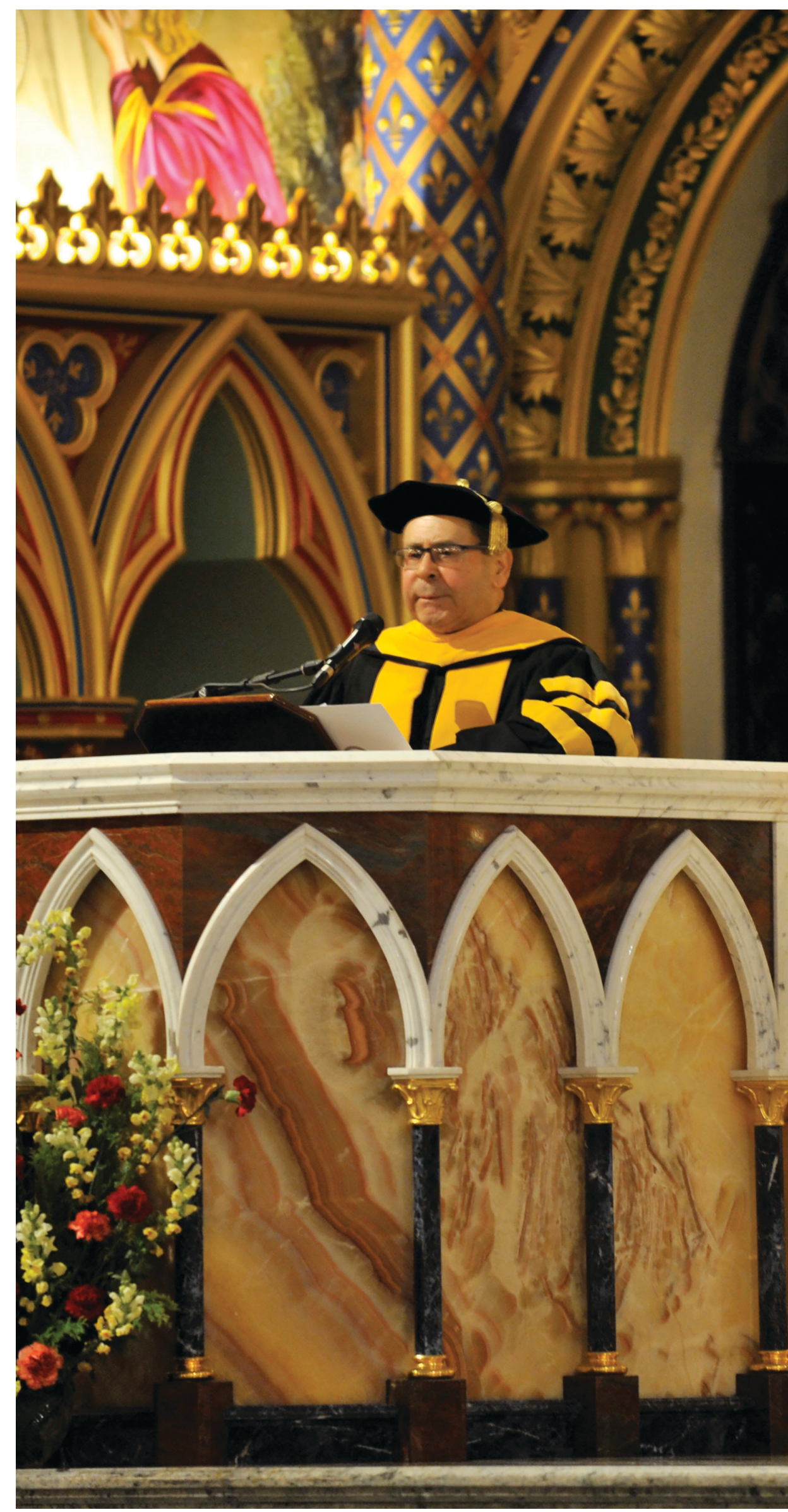
erudito académico y la influencia política de la PUCMM en la realidad nacional” (Ricart. 2008).

\section{Reconocimientos recibidos como académico y ciudadano comprometido}

La labor de monseñor Agripino Núñez Collado ha sido muy bien valorada tanto a nivel local como internacional, haciéndole merecedor de decenas de reconocimientos, por parte de instituciones educativas, gobiernos, organizaciones de la sociedad civil, entre otros.

Más de cuatro décadas al frente de una institución de educación superior avalan sus aportes a la educación Dominicana desde esta Pontificia Universidad Católica Madre y Maestra.

En el discurso que pronunciara el pasado Rector de la Universidad Autónoma de Santo Domingo (UASD), con motivo del Reconocimiento otorgado por esa casa de estudios a la PUCMM en el 50 Aniversario de su fundación, hizo la siguiente afirmación: "La Pontificia Universidad Católica Madre y Maestra y su Rector, monseñor Agripino Núñez Collado, se conjugan en un solo cuerpo que resulta a veces difícil separar. Juntos han aportado a Santiago y al país miles de profesionales y a través de la personalidad carismática y mediadora de monseñor, ambos han jugado un rol importante en varios escenarios y etapas de la vida política y social de la República Dominicana, lo que los ha hecho merecedores del reconocimiento de la sociedad" (Febrillet. 2012).

Resulta difícil enumerar en breves páginas todos los honores otorgados a Agripino Núñez Collado. Presentamos los principales en una apretada síntesis.

Principales honores académicos: Universidad Católica de Fujen, Taipei, Taiwan, República de China, "por su destacado liderazgo educativo desde la Universidad Católica Madre y Maestra, y por sus esfuerzos para fortalecer la amistad y el intercambio entre las Repúblicas de China y Dominicana"; Universidad Autónoma de Guadalajara, México, "por su liderazgo en el campo de la educación y por sus esfuerzos en pro de las relaciones entre las universidades de América Latina"; Universidad de Carolina del Sur, Estados Unidos; "por su ilustrado liderazgo en la educación superior en la República Dominicana e internacionalmente; por sus múltiples contribuciones al avance de su nación a través de la educación; y en reconocimiento a su gran devoción por los más altos ideales académicos"; "Universidad Interamericana (UNICA), República Dominicana, "en reconocimiento a su trayectoria de mediador, conciliador y fomentador del diálogo y la concertación ante los grandes conflictos sociales y políticos que se han producidos en los últimos tiempos en el país" y Profesor Honorario de la Universidad de Arkansas, Little Rock de los Estados Unidos.

Algunos de los reconocimientos de gobiernos nacionales e internacionales: condecoración "Orden Vasco Núñez de Balboa en el Grado de Gran Oficial", por parte del Gobierno de Panamá; "Caballero de la Legión de Honor", otorgada por el gobierno de Francia; "Orden Heráldica de Cristóbal Colón", otorgada por el Gobierno Dominicano; "Encomienda de la Orden del Mérito Civil", otorgada por el Rey de España, Su Majestad Juan Carlos I; "Gran Cruz del Mérito Melitense", otorgada por la Soberana Orden de Malta; "Orden Nacional al Mérito, en el grado de Gran Oficial", de parte del Gobierno del Ecuador; "Orden al Mérito de Duarte, Sánchez y Mella" en el Grado de "Gran Cruz de Plata", conferido por el Gobierno Dominicano; "Condecoración Andrés Bello", de parte del Gobierno de Venezuela; "Orden Nacional Andrés Bello", en la categoría de Primera Clase, de parte del Gobierno de Venezuela; "Oficial de la Orden de las Palmas Académicas", otorgado por el Ministerio Francés de Educación Nacional"; "Grado de Comendador de la Orden de las Artes y las Letras" del Gobierno de Francia y "Palmas Académicas en el Grado de Caballero", conferido por el Gobierno de Francia.

Más recientemente, en la cercanía de su despedida como Rector de la PUCMM, ha sido objeto de varios homenajes, entre los que citamos la Gala de Reconocimientos a Personalidades, realizada por la Junta Central Electoral; la Gala Anual del Consejo Nacional de la Empresa Privada (CONEP) y el Premio de la Academia 2014, otorgado por la Academia Dominicana de la Lengua.

"A monseñor Núñez Collado.., le corresponde el mérito de haber contribuido al desarrollo político, social y económico de la República Dominicana, y haber logrado que la PUCMM sea considerada como la mejor universidad de nuestro país y una de las mejores de Latinoamérica..." (José Darío Suárez Martínez).

Concluimos este artículo citando lo que escribiera Héctor Incháustegui Cabral en el prólogo del libro La UCMM: Un Nuevo Estilo Universitario en la República Dominicana, publicado en el año 1977. Al referirse a la labor de monseñor Núñez en esta Institución, señala que "... es obra de fe, de pasión, de audacia, de dinamismo, de capacidad para poner de su lado a los que saben ayudar y a los que pueden iluminar; una obra, la realizada por monseñor Núñez, que puede apreciarse con los ojos de la cara, por lo que es físicamente hoy la Universidad, y con los ojos del espíritu, porque al espíritu le está reservado ver lo que es grande sin ocupar lugar sobre la tierra". 